\title{
Capecitabine-induced Toxicity: An Outcome Study into Drug Safety
}

Janneke Baan ${ }^{1}$, Monique MEM Bos ${ }^{2 *}$, Savita U Gonesh-Kisoensingh ${ }^{3}$, Iwan A Meynaar ${ }^{4}$, Jelmer Alsma ${ }^{5}$, Erik Meijer ${ }^{6}$ and Arnold GVulto $^{7}$

${ }^{1}$ Albert Schweitzer Hospital, Department of Medicine, Dordrecht, The Netherlands

${ }^{2}$ Reinier de Graaf Group of Hospitals, Department of Medicine, The Netherlands

${ }^{3}$ Saint Lucas Andreas Hospital, Hospital Pharmacy, Amsterdam, The Netherlands

${ }^{4}$ HAGA Hospital, Intensive Care Unit, Den Hague, The Netherlands

${ }^{5}$ Erasmus University Medical Center, department of Internal Medicine, Rotterdam, The Netherlands

${ }^{6}$ Reinier de Graaf Group of Hospitals, Hospital Pharmacy, Delft, The Netherlands

${ }^{7}$ Erasmus University Medical Center, Hospital Pharmacy, Rotterdam, The Netherlands

\begin{abstract}
Background: The use of capecitabine has risen exponentially in the Netherlands since 2001. Clinical trials describe a mild toxicity profile. Because circumstances in daily clinical practice can differ a lot from clinical trial setting, we performed this retrospective analysis in a large community hospital to verify toxicity in a clinical situation.

Methods: A retrospective cohort study was conducted in patients with malignancies of the gastrointestinal tract or breast treated with capecitabine in the period of January 2007 to January 2009. Primary study endpoint was the incidence and severity of capecitabine-induced toxicity in daily clinical practice. Secondary endpoint concerned determination of risk factors for toxicity due to capecitabine.
\end{abstract}

Results: Of 281 patients $92 \%$ experienced some degree of toxicity. Grade 3-4 toxicity occurred in $30 \%$ of patients receiving monotherapy and in $47 \%$ with combination therapy. This was in accordance with the literature. Type of toxicity varied, but gastro intestinal symptoms and hand foot syndrome were most commonly found. Risk of toxicity increased with increasing age, independently of creatinine clearance.

Conclusions: Therapy with capecitabine monotherapy or capecitabine containing regiments in daily clinical practice is accompanied by considerable toxicity, but frequency and severity are consistent with published clinical trials. More toxicity can be expected with increasing age.

\section{Keywords: Cancer; Capecitabine; Chemotherapy; Toxicity}

Abbreviations: 5-FU: Fluorouracil; TS: Thymidylate Synthase; GI: Gastrointestinal; DPD: Dihydropyrimidine Dehydrogenase; HFS: Hand Food Syndrome; BMS: Bone Marrow Suppression; DBC: Diagnosis Treatment Combination; CapOx: Capeciatabine and Oxaliplatin; ECC: Epirubicin, Cisplatin and Capecitabine; EOX: Epirubicin, Oxaliplatin and Capecitabine; CapIri: Capecitabine and Irinotecan; PNP: Polyneuropathy; TEE: Thrombo Embolic Events

\section{Introduction}

Capecitabine $\left(\mathrm{Xeloda}^{\circledR}\right)$ is an oral prodrug of Fluorouracil (5FU) and therefore a Thymidylate Synthase inhibitor (TS inhibitor). It is widely used in the treatment of patients with malignancies of the Gastrointestinal (GI) tract and the breast. It can be prescribed as monotherapy or in combination with other drugs such as oxaliplatin, irinotecan and cisplatin for GI cancer, and a taxane or monoclonal antibodies such as trastuzumab (Herceptin ${ }^{\circledR}$ ) or bevacizumab $\left(\right.$ Avastin $\left.^{\circledR}\right)$ for breast cancer [1-4]. Since its introduction the number of capecitabine prescriptions in the Netherlands has risen exponentially from 300 in 2001 to 58,200 in 2008 (Dutch Foundation for Pharmaceutical Statistics).

Metabolism of capecitabine to the active compound 5-FU is conducted in three activation steps. Thymidine phosphorylase is the enzyme responsible for the final step, resulting in conversion to 5-FU. This enzyme has a 3.2 times higher concentration in various solid tumours compared with normal adjacent tissue theoretically giving selective activation of the drug and less systemic toxicity [5]. The Dihydropyrimidine Dehydrogenase (DPD) is the pivotal enzyme in detoxification and elimination of 5-FU in humans. Patients with DPD deficiency are therefore at increased risk of 5-FU toxicity and consequently capecitabine toxicity. Frequency of DPD deficiency ranges from $3-5 \%$ in the Caucasian population $[6,7]$.

The advantage of capecitabine over most other TS inhibitors is its oral use in an outpatient setting. The other oral fluoropyrimidine S-1 has a comparable efficacy and safety profile, but is predominantly prescribed in Asia [8]. According to the product characteristics, the overall safety profile of capecitabine is comparable with several other chemotherapy regimens for the indications mentioned. Most common side effects are mild diarrhoea, stomatitis, Hand Food Syndrome (HFS) and fatigue. The severity of adverse events is related to combination treatment, creatinine clearance and age. Dose adjustments are indicated in patients with a creatinine clearance beneath $50 \mathrm{ml} / \mathrm{min}$ and might be indicated in patients of 60 years and older. Absolute contraindications are known DPD deficiency, renal impairment (creatinine clearance beneath $30 \mathrm{ml} / \mathrm{min}$ ), severe hepatic impairment and severe Bone Marrow Suppression (BMS). Doses range from $1250-2500 \mathrm{mg} / \mathrm{m}^{2}$ a day, according to disease-specific chemotherapy protocols. (http:// eudrapharm.eu. January 2009).

*Corresponding author: Monique MEM Bos, Reinier de Graaf Group of Hospitals, Reinier de Graaf weg 3-11, 2625 AD Delft, The Netherlands, Tel: 0031-152603165/3101; Fax: 0031-15-2603116; E-mail: Bos@rdgg.nl

Received January 16, 2014; Accepted February 25, 2014; Published March 05 , 2014

Citation: Baan J, Bos MMEM, Gonesh-Kisoensingh SU, Meynaar IA, Alsma J, et al. (2014) Capecitabine-induced Toxicity: An Outcome Study into Drug Safety. J Integr Oncol 3: 113 doi:10.4172/2329-6771.1000113

Copyright: (c) 2014 Baan J, et al. This is an open-access article distributed under the terms of the Creative Commons Attribution License, which permits unrestricted use, distribution, and reproduction in any medium, provided the original author and source are credited. 
While clinical trials describe a mild toxicity profile, study populations differ from those encountered in daily clinical practice. This study was designed to measure toxicity in a clinical cancer population in a large community hospital the Netherlands to determine if results were comparable with those from registration studies or if toxicity was higher than suggested by the literature whereas the general opinion is that oral cytostatic therapy has a relatively mild toxicity-profile. As a result the main goal of this study was to evaluate toxicity of capecitabine containing chemotherapeutic regimens, including the consequences of hospital admission, independent of efficacy or other clinical outcome.

\section{Methods}

\section{Study design}

We conducted a retrospective cohort study. The regional medical ethics committee of South West Netherlands was informed. Approval from patients or relatives was not necessary because data gathering was anonymous. A note was made in each medical chart that information was used for medical research.

\section{Setting and patients}

The study was conducted in the oncology department of a community teaching hospital in the Netherlands covering a population of 350,000 inhabitants. Each patient was evaluated by a medical oncologist in the outpatient clinic before commencing capecitabinebased treatment. Indication for treatment and known adverse events were discussed with the patient and relatives. In a second separate visit the patient was extensively informed by a nursing specialist. A written patient protocol was provided including telephone numbers of the nursing staff and oncology unit, which can be contacted at all times. All patients aged 18 years and up with malignancies of the GI tract or breast, treated with capecitabine in mono- or combination therapy in the period of January 2007 to January 2009 were included. Toxicity assessments were performed on the day before the next course of chemotherapy by a clinical oncologist or an oncology nurse specialist. According to the local protocols toxicity was scored according to the CTC-toxicity criteria, focusing especially on general complaints as fatigue and loss of appetite, combined with special symptoms as diarrhoea, nausea and vomiting, neuropathies and Hand \& Foot syndrome

The diagnosis was recorded for all patients in the diagnosis treatment combination system (www.cz.nl) in the Netherlands and this was used to identify all patients treated for colon cancer, oesophageal cancer, stomach cancer and breast cancer. The patients treated with capecitabine were retrieved using the electronic personal health record. In patients with metastatic breast cancer capecitabine was subscribed as monotherapy or sometimes in combination with docetaxel as a palliative treatment. Upper GI tract tumours were either treated with Epirubicin, Cisplatin and Capecitabine (ECC) or Epirubicin, Oxaliplatin and Capecitabine (EOX). Capecitabine was combined with Oxaliplatin (CapOx) or Irinotecan (CapIri) for colorectal cancer. Dose reduction to $75 \%$ of the original capecitabine dose at the start of chemotherapy was given to those with a creatinine clearance between $30-50 \mathrm{ml} / \mathrm{min}$. Other factors that sometimes led to dose reductions were age and previous toxicity judged by the prescribing oncologist.

Patient data was collected using electronic and paper medical charts. Possible capecitabine-related toxicity and other characteristics were extracted. We assessed the degree of toxicity according to the National Cancer Institute Common Terminology Criteria for Adverse Events (CTC-AE) version 4.0. Baseline characteristics, serum creatinine, comorbidity, use of other medicine(s), type of cancer, stage of cancer, setting of treatment, type and daily dose of chemotherapy. Information on dose adjustments and hospital admissions was also registered. Creatinine clearance was calculated for all patients using the Cockcroft and Gault formula. Body mass index and body surface area were calculated using bodyweight and height measured at start. All data were entered anonymously in the database.

\section{Outcomes and literature review}

Primary endpoint was the incidence and severity of observed capecitabine-induced toxicity in daily practice. Toxicity frequencies were described separately for mono- and combination therapy. Secondary endpoint was the determination of risk factors for toxicity, using gender, age, and creatinine clearance, dose adjustment at start and type of chemotherapy. For comparison of the outcomes of this study with the frequency of toxicity in pivotal trials, a literature review was conducted using the PubMed database. A separate search was performed for each chemotherapeutic regimen. Articles were selected using the following criteria: similar dose intensities as used in our hospital, comparable indications of chemotherapeutic agent, group size and extensive information on the safety profile must be available [917]. Search for: capecitabine AND toxicity AND colorectal cancer OR breast cancer AND limits; clinical trial phase II, III or IV and English.

\section{Statistical analysis}

All analyses were performed with SPSS for Windows, version 16, (SPSS, Chicago, II, USA). To compare baseline characteristics the chi square test was used in categorical data and the t-test was used in continuous variables with a normal distribution. A multivariate regression model was used to demonstrate whether any characteristics were present that affect toxicity. Variables used in this model were; gender, age, tumour group, metastases, treatment setting, mono- or combination therapy, dose, creatinine clearance at start and body surface area. A p-value $<0.05$ was considered significant.

\section{Results}

Between January 2007 and January 2009, a total of 570 patients was retrieved carrying a diagnosis of gastro-intestinal or breast cancer. Two hundred eighty one of them had been treated with capecitabine and were included in this analysis. Most patients had a Caucasian phenotype, as to be expected in the outer skirts of the Netherlands. Baseline characteristics are listed in Table 1. Mean age was 64 years (SD 10), 46.9\% of the patients were female. Predominant diagnosis was colorectal cancer $(59.0 \%)$ while $26.7 \%$ had cancer of the upper GI tract and $13.9 \%$ had breast cancer. The majority of the patients (61.9\%) suffered from metastastatic disease undergoing chemotherapy in a palliative setting. Most patients (86.8\%) underwent combination therapy of capecitabine with oxaliplatin, epirubicin, cisplatin, irinotecan or a taxane. Monotherapy capecitabine was only subscribed in $13.2 \%$. Two third of the breast cancer patients were treated with single agent capecitabine. ECC was the predominant combination in upper GI tract cancer and CapOx in colorectal cancer patients while only a small group was treated with monotherapy capecitabine or a combination with irinotecan. According to treatment protocols the dose should have been reduced to $75 \%$ of the original dose in every patient with a creatinine clearance of $30-50 \mathrm{ml} / \mathrm{min}(\mathrm{n}=19)$. Protocol was followed in $69 \%$ of these patients. In patients with a creatinine clearance over $50 \mathrm{ml} / \mathrm{min}(\mathrm{n}=262)$ the dose was reduced in $9 \%$, mostly based on clinical condition or co-morbidity of the patient as judged by the treating oncologist. 


\begin{tabular}{|c|c|c|}
\hline \multirow[b]{2}{*}{ Patient characteristics } & \multicolumn{2}{|c|}{ All patients $n=281$} \\
\hline & & \\
\hline Mean age in years $\left(\mathrm{SD}^{1}\right)$ & 64 & $(10)$ \\
\hline Female (\%) & 132 & $(46.9)$ \\
\hline Mean Body Surface Area (SD) & 1.9 & $(0.2)$ \\
\hline Mean Cockcroft clearance in $\mathrm{ml} / \mathrm{min}(\mathrm{SD})$ & 84 & $(25)$ \\
\hline Creatinine clearance of $30-50 \mathrm{ml} / \mathrm{min}(\%)$ & 19 & $(6.7)$ \\
\hline \multicolumn{3}{|l|}{ Diagnosis } \\
\hline Colorectal cancer (\%) & 166 & $(59.0)$ \\
\hline Upper gastrointestinal tract cancer ${ }^{2}(\%)$ & 75 & $(26.7)$ \\
\hline Breast cancer $(\%)$ & 39 & $(13.9)$ \\
\hline Metastases (\%) & 174 & (61.9) \\
\hline Non- metastatic cancer (\%) & 107 & $(38.0)$ \\
\hline \multicolumn{3}{|l|}{ Treatment setting } \\
\hline Mean Daily Dose (mg/day) (SD) & 1917 & $(277)$ \\
\hline Neo-adjuvant (\%) & 56 & (19.9) \\
\hline Adjuvant (\%) & 85 & $(30.2)$ \\
\hline Palliative (\%) & 140 & $(49.8)$ \\
\hline Monotherapy Capecitabine ${ }^{3}(\%)$ & 37 & $(13.2)$ \\
\hline Colorectal cancer & 9 & $(3.2)$ \\
\hline Breast cancer & 28 & $(10.0)$ \\
\hline Combination therapy (\%) & 244 & $(86.8)$ \\
\hline \multicolumn{3}{|l|}{ Colorectal cancer } \\
\hline - CapOx ${ }^{4}$ & 133 & $(47.3)$ \\
\hline - Caplri ${ }^{5}$ & 24 & $(8.5)$ \\
\hline \multicolumn{3}{|l|}{ Upper gastro-intestinal cancer } \\
\hline$-\mathrm{ECC}^{6}$ & 66 & (23.5) \\
\hline - EOX & 9 & $(3.2)$ \\
\hline \multicolumn{3}{|l|}{ Breast cancer } \\
\hline - Capecitabine/Docetaxel ${ }^{8}$ & 11 & (3.9) \\
\hline
\end{tabular}

${ }^{1} \mathrm{SD}$ : standard deviation, ${ }^{2}$ Represents oesophageal cancer, gastric cancer and cancer of the gastro-intestinal junction, ${ }^{3}$ Capecitabine $2500 \mathrm{mg} / \mathrm{m}^{2}$ day $1-14 \mathrm{q} 21$ days, ${ }^{4}$ Capecitabine $2000 \mathrm{mg} / \mathrm{m}^{2}$ day $1-14$ in combination with oxaliplatin $135 \mathrm{mg} / \mathrm{m}^{2}$ day 1 q 21 days, ${ }^{5}$ Capecitabine $2000 \mathrm{mg} / \mathrm{m}^{2}$ day $1-14$ in combination with irinotecan $250 \mathrm{mg} / \mathrm{m}^{2}$ day $1 \mathrm{q} 21$ days, ${ }^{6}$ Epirubicine $50 \mathrm{mg} / \mathrm{m}^{2}$, cisplatin $60 \mathrm{mg} / \mathrm{m}^{2}$ day 1 in combination with Capecitabine $2000 \mathrm{mg} / \mathrm{m}^{2}$ day $1-14$ q 21 days, ${ }^{7}$ Epirubicine 50 $\mathrm{mg} / \mathrm{m}^{2}$, oxaliplatin $130 \mathrm{mg} / \mathrm{m}^{2}$ day 1 in combination with capecitabine $1350 \mathrm{mg} / \mathrm{m}^{2}$ daily q 21 days, ${ }^{8}$ Capecitabine $2000 \mathrm{mg} / \mathrm{m}^{2}$ day $1-14$ and docetaxel $75 \mathrm{mg} / \mathrm{m}^{2}$ day 1 , q 21 days.

Table 1: Patient characteristics, diagnosis and treatment at date of start chemotherapy.

Of the 281 patients analysed, 258 patients reported toxicity (91.8\%). The all-grade toxicity reported most often was Polyneuropathy (PNP) by 112 patients $(39.8 \%)$, followed by HFS in 83 patients $(29.5 \%)$ and nausea/vomiting in 54 patients (19.2\%) (Table 2).

\section{Monotherapy}

Ten of the 37 patients (27.0\%) had grade 3 toxicity, one showed grade 4 toxicity and one patient died of mucositis (DPD deficiency was confirmed afterwards). The incidence of HFS and GI symptoms was high in all grades. Most of the patients suffering grade 3 toxicity had gastrointestinal toxicity, mainly a combination of diarrhoea, abdominal cramps, vomiting and stomatitis. Other grade 3 toxicity comprised BMS and HFS. No cardiac toxicity or Thromboembolic Events (TEE) was mentioned. Hospital admission due to toxicity was necessary in 7 patients (16.2\%), mainly due to mucositis. Reasons to stop chemotherapy were disease progression or toxicity. Only 5 (13.5\%) patients completed the planned cycles.

\section{Combination therapy}

In the combination therapy group of 244 patients, $3(1.2 \%)$ died due to toxicity (unfortunately not tested for DPD deficiency) and 110 (45.0\%) had grade 3-4 toxicity. When comparing the mono- and combination therapy groups, significantly higher incidences of HFS and PNP were found in the combination therapy group (Table 2), the latter one mainly in relation with oxaliplatin. Grade 3-4 toxicity was frequent and varied. Most prominent was some form of mucositis in 31 patients (12.7\%); 4 out of 6 patients tested had a DPD deficiency, but no one of them died. Other grade 3-4 adverse events were HFS (8.3\%), PNP (4.9\%), BMS (5.3\%), TEE (3.1\%) and cardiac events (2.6\%). Hospital admittance occurred in $23.3 \%$ because of mucositis. Reasons to stop chemotherapy were disease progression, toxicity and completing the planned cycles.

Significantly less toxicity was observed in patients receiving monotherapy as compared to the patients receiving combination therapy: out of 37 patients receiving monotherapy 8 (21.6\%) experienced no toxicity, whereas out of 244 patients receiving combination therapy $15(6.1 \%)$ experienced no toxicity, $(p=0.015$, $\mathrm{Chi}^{2}$ test (Table 2)). No significant difference in toxicity was present in the separate malignancies. In Table 3 the results of our analysis is compared with the reference literature for the various types of toxicity.

In the regression model used to describe determinants that affect toxicity, significantly more patients experienced a worse grade of toxicity in the combination therapy group $(\mathrm{p}=0.023)$. A trend was seen of more and greater toxicity with increasing age $(\mathrm{p}=0.055)$ (Table 4$)$.

\section{Discussion}

In this single-centre retrospective cohort study we found that toxicity from capecitabine is reported frequently: only $8 \%$ experienced no toxicity and almost half of the patients (44\%) reported Grade 3-4 toxicity. One third of patients treated with capecitabine, whether or not in combination with other chemotherapeutic agents, discontinued chemotherapy as a result of toxicity. Although impressive, this is comparable with other publications.

When comparing results with the literature (Table 3 ), the main difference noticed was the frequency of GI symptoms. Mucositis is frequent, but often poorly described in the literature. As previously mentioned, it mainly consists of diarrhoea, abdominal cramps, vomiting and stomatitis. When combining the frequencies of those

\begin{tabular}{|c|c|c|c|c|c|c|c|}
\hline \multirow[b]{2}{*}{ No toxicity } & \multicolumn{2}{|c|}{$\begin{array}{l}\text { All patients } \\
n=281(\%)\end{array}$} & \multicolumn{2}{|c|}{$\begin{array}{l}\text { Monotherapy } \\
\text { capecitabine } \\
n=37(\%)\end{array}$} & \multicolumn{2}{|c|}{$\begin{array}{l}\text { Capecitabine } \\
\text { combined with } \\
\text { other chemotherapy }{ }^{1} \\
n=244(\%)\end{array}$} & \multirow{2}{*}{\begin{tabular}{|l} 
p-value \\
\\
0.015
\end{tabular}} \\
\hline & 23 & $(8.2)$ & 8 & $(21.6)$ & 15 & $(6.1)$ & \\
\hline Grade 1 & 172 & (61.2) & 18 & $(48.6)$ & 148 & (60.6) & ns \\
\hline Grade 2 & 168 & $(59.8)$ & 21 & $(56.7)$ & 145 & $(59.4)$ & ns \\
\hline Grade 3 & 106 & (37.7) & 10 & $(27.0)$ & 96 & (39.3) & ns \\
\hline Grade 4 & 26 & $(9.3)$ & 1 & $(2.7)$ & 25 & $(10.2)$ & ns \\
\hline Grade 5 & 4 & $(1.4)$ & 1 & $(2.7)$ & 3 & $(1.2)$ & ns \\
\hline $\begin{array}{l}\text { No or mild } \\
\text { toxicity } \\
(\leq \text { Grade } 2)\end{array}$ & 157 & (55.9) & 33 & $(89.1)$ & 124 & $(50.8)$ & 0.03 \\
\hline $\begin{array}{l}\text { Severe toxicity } \\
(\geq \text { Grade } 3)\end{array}$ & 124 & $(44.1)$ & 14 & $(37.8)$ & 110 & $(45.0)$ & 0.03 \\
\hline $\begin{array}{l}\text { Hospital } \\
\text { admission due } \\
\text { to toxicity }\end{array}$ & 64 & $(22.8)$ & 7 & $(16.2)$ & 57 & (23.3) & ns \\
\hline
\end{tabular}

${ }^{1}$ Capecitabine in combination with oxaliplatin, irinotecan, epirubicine and cisplatin, epirubicine and oxaliplatin or with docetaxel, ${ }^{2}$ Comparing symptoms and toxicity for monotherapy with combination therapy.

Table 2: Toxicity grades reported by all patients and separately for those receiving either monotherapy or combination therapy. 


\begin{tabular}{|l|l|l|l|l|}
\hline \multicolumn{4}{|l|}{ Monotherapy capecitabine } & \multicolumn{2}{l|}{ Combination therapy } \\
\hline & $\begin{array}{l}\text { Toxicity in this } \\
\text { study (\%) }\end{array}$ & $\begin{array}{l}\text { Toxicity in } \\
\text { literature (\%) }\end{array}$ & $\begin{array}{l}\text { Toxicity in this } \\
\text { results (\%) }\end{array}$ & $\begin{array}{l}\text { Toxicity in } \\
\text { literature (\%) }\end{array}$ \\
\hline Nausea/Vomiting & 16.2 & $3-35$ & 19.6 & $6.0-45.9$ \\
\hline $\begin{array}{l}\text { Mucositis } \\
\text { (e.g.stomatistis, } \\
\text { gastritis) }\end{array}$ & 27.0 & $2-22$ & 17.2 & $14.4-71.7$ \\
\hline Diarrhea & 24.3 & $0-30$ & 14.3 & $5.1-27.0$ \\
\hline $\begin{array}{l}\text { Hand and foot } \\
\text { syndrome }\end{array}$ & 10.8 & 17.1 & 25.0 & $36.6-83.7$ \\
\hline $\begin{array}{l}\text { Polyneuropathy } \\
\text { none marrow }\end{array}$ & 8.1 & n.d.a. & 44.3 & $17.0-64.2$ \\
\hline $\begin{array}{l}\text { Bonpression } \\
\text { suppren }\end{array}$ & $0-9$ & 15.6 & $7.5-72.0$ \\
\hline $\begin{array}{l}\text { Thrombo embolic } \\
\text { event }\end{array}$ & 2.7 & n.d.a. & 8.6 & n.d.a. \\
\hline Cardiac event & 0.0 & n.d.a. & 4.9 & n.d.a. \\
\hline
\end{tabular}

n.d.a.: no data available. Results are combined for the different types of toxicity, highest and lowest percentage are given extracted from the different publications. (O'Shaughnessy et al. [16], Scheithauer et al. [12], Zeuli et al. [13], Bajetta et al. [18], Batista et al. [15], Ajani [3], Cunningham et al. [14])

Table 3: Frequency of different types of toxicity as compared to the reference literature, separately for mono-and combination therapy.

\begin{tabular}{|l|l|l|l|}
\hline & p-value & Odds Ratio & $\begin{array}{l}\text { 95\% confidence } \\
\text { interval }\end{array}$ \\
\hline Age at start chemotherapy & 0.055 & 1.024 & $0.99-1.049$ \\
\hline $\begin{array}{l}\text { Monotherapy vs. combination } \\
\text { chemotherapy }\end{array}$ & 0.023 & 0.404 & $0.186-0.880$ \\
\hline Female sex vs. male sex & 0.2 & 1.4 & $0.9-2.3$ \\
\hline Body surface area & 0.6 & 1.6 & $0.3-7.6$ \\
\hline Creatinine clearance & 0.3 & 1.0 & $1.0-1.0$ \\
\hline Metastases & 0.4 & 1.2 & $0.7-2.0$ \\
\hline Neo-adjuvant chemotherapy & 0.1 & 0.5 & $0.2-1.1$ \\
\hline Adjuvant chemotherapy & 0.5 & 0.8 & $0.3-1.7$ \\
\hline Dose adjustment & 0.9 & 1.0 & $1.0-1.0$ \\
\hline Colorectal cancer & 0.9 & 1.0 & $0.4-2.7$ \\
\hline Upper gastrointestinal tract cancer & 0.3 & 1.9 & $0.6-5.7$ \\
\hline
\end{tabular}

Variables used in this model were; gender, age at start chemotherapy, tumor group, metastases, setting of treatment, mono- or combination therapy, dose, creatinine clearance and body surface area at start chemo therapy. p-value of $<0.05$ was considered significant.

Table 4: Mmultivariate regression analysis of different characteristics that could influence toxicity.

different variables in the literature and comparing them with the frequency of mucositis recognised in our study, they fell into the same range. In all groups grade 3-4 BMS was less frequent as is mentioned in the literature, possible related to the strict in hospital protocol guidelines, not allowing to start the next palliative cycle of cytostatics without full recovery of the cell counts. And, importantly the several phases two and three prospective clinical trial used as reference are more detailed in registering toxicity including BMS. Overall a low frequency of significant HFS was present compared to the literature. HFS might be underscored because it can diminish in the capecitabinefree week before starting a new cycle making it difficult to estimate the right grade of toxicity. In the German multicentre analysis of Hofheinz 29.3\% developed any HFS suggesting an association of HFS and survival and a higher probability of developing any-grade gastrointestinal toxicity in patients with colorectal cancer $[17,18]$. Our data could not confirm such a relationship, probably due to a smaller sample size. Cardiotoxicity was not found in the monotherapy group, combination therapy showed $4.9 \%$ all-grade toxicity and $2.6 \%$ grade 3-4. Van Cutsum reported 5 cases of cardiotoxicity in a total group of 108 patients, spread over three treatment groups containing

capecitabine [11]. In several reviews a frequencies in a range of 3-6\% of all grades of cardiotoxicity in patients treated with capecitabine $[19,20]$. Our data are comparable.

It must be noted that monotherapy was easier to compare with the literature than the combination therapy group. For the latter it is more useful to look at the individual cytostatics, used in the different regiments, as they give different types of toxicity. In the study group patient numbers within these different sorts of chemotherapeutic regimens were too small to compare with the literature. Even so the frequencies in the overall group of combination therapy still fell in the range mentioned in the literature.

Hospital admission because of toxicity in clinical trials ranges from $12-20 \%$ in single agent capecitabine and $14-28 \%$ in combination chemotherapy $[8,9,12,15]$. Rate of admittance in our analysis was as could be expected according these publications.

The secondary endpoints, describing possible risk factors other than creatinine clearance beneath $50 \mathrm{ml} / \mathrm{min}$, showed a significant trend towards more toxicity with advancing age. Creatinine clearance could be a confounding factor, however in both groups no significant correlation was found between creatinine clearance and toxicity. This is as we expected, because the dose of chemotherapy was adjusted for creatinine clearance, making both groups similarly prone to toxicity. As to be expected older patients have more comorbidity resulting in a worse condition compared with younger patients and there for having a higher chance of toxicity. They are also more prone to take medicines wrongly, for example by non-compliance with the medicine preventing adverse events, or taking more chemotherapy pills then necessary [21]. Apart from this elderly patients can report toxicity more frequently [22].

We recognize several limitations of our study. First, this is a retrospective analysis. As such, documentation can vary depending on severity of events and can differ between oncologists. This may have led to an underestimation of the frequencies of toxicity, especially grade one and two toxicity, not directly relevant to clinical use. Another point to consider is that the patient compliance was not reported, but this is a known problem in patients using oral medicines. This could affect the number and frequency of the side effects. Improper use of medicines, e.g. continuing the drugs in the stop week can give an overestimation of toxicity. Not reporting symptoms of toxicity when visiting the outpatient clinic could lead to an underestimation of toxicity; however the staff of the oncology unit was easily accessible day and night and the nursing specialists tended to check on the patients by telephone in the second week taking capecitabine. Thirdly, only a minority of patients received monotherapy with capecitabine, and toxicity in patients with combination therapy are probably related to the an additive or synergistic effect of capecitabine and the other components of the treatment. For example is the CapIri regiment not often used anymore due to the known synergistic toxicity of both cytostatic drugs, especially diarrhoea. A particular strength of this study is that to our knowledge this is the only retrospective analysis where toxicity of capecitabine as used in daily practice has been directly compared with the known toxicity from the literature. Although the groups of the individual combinations with capecitabine were relatively small it is unlikely that new insights into drug toxicity of capecitabine are to be found when the volume of patients is higher.

\section{Conclusions}

There is a high frequency of toxicity in patients receiving 
Citation: Baan J, Bos MMEM, Gonesh-Kisoensingh SU, Meynaar IA, Alsma J, et al. (2014) Capecitabine-induced Toxicity: An Outcome Study into Drug Safety. J Integr Oncol 3: 113 doi:10.4172/2329-6771.1000113

capecitabine monotherapy in clinical practice, but these numbers are comparable with the frequencies described in clinical trials. Although the combination therapy group was more difficult to compare, toxicity levels also seemed comparable with the literature. Capecitabine creates a frequent need for hospital admission and high frequency in stopping because of toxicity. The seriousness of these side effects should not be underestimated, and need appropriate anticipation. Elderly patients are more prone to experiencing toxicity, independently of renal function. More research should be conducted to minimize adverse events in this vulnerable group of patients.

\section{Acknowledgements}

The authors wish to express their appreciation and gratitude to Wim Hop for his statistical guiding and Judith Martin for her excellent editor support.

\section{References}

1. Ershler WB (2006) Capecitabine monotherapy: safe and effective treatment for metastatic breast cancer. Oncologist 11: 325-335.

2. Schmoll HJ, Arnold D (2006) Update on capecitabine in colorectal cancer Oncologist 11: 1003-1009.

3. Ajani J (2006) Review of capecitabine as oral treatment of gastric gastroesophageal, and esophageal cancers. Cancer 107: 221-231.

4. Tripathy D (2007) Capecitabine in combination with novel targeted agents in the management of metastatic breast cancer: underlying rationale and results of clinical trials. Oncologist 12: 375-389.

5. Schüller J, Cassidy J, Dumont E, Roos B, Durston S, et al. (2000) Preferentia activation of capecitabine in tumor following oral administration to colorectal cancer patients. Cancer Chemother Pharmacol 45: 291-297.

6. Morel A, Boisdron-Celle M, Fey L, Soulie P, Craipeau MC, et al. (2006) Clinica relevance of different dihydropyrimidine dehydrogenase gene single nucleotide polymorphisms on 5-fluorouracil tolerance. Mol Cancer Ther 5: 2895-2904.

7. Mattison LK, Fourie J, Desmond RA, ModakA, Saiff MW, et al. (2006) Increased Prevalence of Dihydropyrimidine Dehydrogenase Deficiency in AfricanAmericans Compared with Caucasians. Clin Cancer Res 12: 5491-5495.

8. Zhang X, Cao C, Zhang Q, Chen Y, Gu D, et al. (2014) Comparison of the efficacy and safety of S-1-based and capecitabine-based regimens in gastrointestinal cancer: a meta-analysis. PLoS One 9: e84230.

9. Blum JL, Jones SE, Buzdar AU, LoRusso PM, Kuter I, et al. (1999) Multicenter phase II study of capecitabine in paclitaxel-refractory metastatic breast cancer. J Clin Oncol 17: 485-493.
10. Blum JL, Dieras V, Lo Russo PM, Horton J, Rutman O, et al. (2001) Multicenter, Phase II study of capecitabine in taxane-pretreated metastatic breast carcinoma patients. Cancer 92: 1759-1768.

11. Cutsem Van E, Findlay M, Osterwalder B, Kocha W, Dalley D, et al. (2000) Capecitabine, an oral fluoropyrimidine carbamate with substantial activity in advanced colorectal cancer: results of a randomized phase II study. J Clin Oncol 18: 1337-1345.

12. Scheithauer W, Kornek GV, Raderer M, Schüll B, Schmid K, et al. (2003) Randomized multicenter phase II trial of two different schedules of capecitabine plus oxaliplatin as first-line treatment in advanced colorectal cancer. J Clin Oncol 21: 1307-1312.

13. Zeuli M, Nardoni C, Pino MS, Gamucci T, Gabriele A, et al. (2003) Phase II study of capecitabine and oxaliplatin as first-line treatment in advanced colorectal cancer. Ann Oncol 14: 1378-1382.

14. Cunningham D, Starling N, Rao S, Iveson T, Nicolson M, et al. (2008) Capecitabine and oxaliplatin for advanced esophagogastric cancer. $\mathrm{N}$ Engl J Med 358: 36-46.

15. Batista N, Perez-Manga G, Constenla M, Ruiz A, Carabantes F, et al. (2004) Phase II study of capecitabine in combination with paclitaxel in patients with anthracycline-pretreated advanced/metastatic breast cancer. $\mathrm{Br} \mathrm{J}$ Cancer 90 1740-1746.

16. O'Shaughnessy J, Miles D, Vukelja, S, Moiseyenko V, Ayoub JP, et al. (2002) Superior survival with capecitabine plus docetaxel combination therapy in anthracycline-pretreated patients with advanced breast cancer: phase III trial results. J Clin Oncol 20: 2812-2823.

17. Hofheinz RD, Heinemann V, von Weikersthal LF, Laubender RP, Gencer D, et al. (2012) Capecitabine-associated hand-foot-skin reaction is an independent clinical predictor of improved survival in patients with colorectal cancer. $\mathrm{Br} \mathrm{J}$ Cancer 107: 1678-1683.

18. Bajetta E, Di Bartolomeo M, Mariani L, Cassata A, Artale S, et al. (2004) Randomized multicenter Phase II trial of two different schedules of irinotecan combined with capecitabine as first-line treatment in metastatic colorectal carcinoma. Cancer 100: 279-287.

19. Walko CM, Lindley C (2005) Capecitabine: a review. Clin Ther 27: 23-44.

20. Saif MW, Katirtzoglou NA, Syrigos KN (2008) Capecitabine: an overview of the side effects and their management. Anticancer Drugs 19: 447-464.

21. Banning M (2008) Older people and adherence with medication: a review of the literature. Int J Nurs Stud 45: 1550-1561.

22. Regnier Denois V, Poirson J, Nourissat A, Jacquin JP, Guastalla JP, et al. (2011) Adherence with oral chemotherapy: results from a qualitative study of the behaviour and representations of patients and oncologists. Eur $\mathrm{J}$ Cancer Care (Engl) 20: 520-527. 